Bol. Acad. peru. leng. 67. 2020 (205-220)

\title{
CAMBIOS SEMÁNTICOS EN EL LÉXICO LUNQU DEL ANEXO DE TUCTUMPAYA
}

\section{SEMANTIC CHANGES IN THE LUNQU LEXICON OF THE TUCTUMPAYA APPENDIX}

\author{
Yazmine Farfán Cayllahua \\ Universidad Nacional Mayor de San Marcos \\ https://orcid.org/0000-0003-2521-5529 \\ Gionella Vilca Coaguila \\ Universidad Nacional Mayor de San Marcos \\ https://orcid.org/0000-0002-9836-9660
}

\section{Resumen:}

El LÉXICO LUNQU es hablado por los lunqus 'campesinos' de las campiñas arequipeñas y, como es una lengua especial, solo es entendida por ellos. Este léxico presenta influencia de lenguas amerindias, tanto del quechua como del aimara, pero en el presente trabajo, solo se estudian las entradas que son préstamos del quechua y que conservan intacta la palabra base. Como las palabras seleccionadas muestran cambios en su información semántica, se plasman los rasgos semánticos característicos de la palabra y se hace una breve explicación del cambio semántico producido en dicho léxico.

Palabras clave: lunqu, cambios semánticos, léxico, contacto lingüístico, castellano andino. 


\section{Abstract:}

The Lunqu lexicon is spoken by the Lunqus 'peasants' of the Arequipa countryside and, as it is a special language, it is only understood by them. This lexicon is influenced by Amerindian languages, both Quechua and Aymara, but in this paper, we only study those entries borrowed from Quechua and that keep the base word intact. Since the selected words show changes in their semantic information, the characteristic semantic features of the word are shown and a brief explanation about the semantic change produced in that lexicon is given.

Key words: Lunqu, semantic changes, lexicon, linguistic contact, Andean Spanish.

Fecha de recepción: $\quad 10 / 01 / 2020$

Fecha de aceptación: $\quad$ 25/03/2020

\section{Introducción}

La presente investigación, "Cambios semánticos en el léxico lunqu del anexo de Tuctumpaya, Arequipa», es un trabajo orientado al estudio del cambio semántico en el léxico usado por los autodenominados lunqus, los cuales suelen vivir en las campiñas arequipeñas, entre las más importantes se encuentran Characato, Tiabaya, Sabandía, Paucarpata, Cerro Colorado. Este trabajo busca revalorizar el léxico especial usado por los arequipeños; sin embargo, al momento de buscar tanto en el Diccionario de americanismos (2010) como en el Diccionario de lengua española (2014), estas no se encuentran con las voces que se proponen en el presente estudio.

En el LÉXICO LUNQU se presentan definiciones de las palabras usadas en el léxico coloquial de los habitantes de las campiñas arequipeñas. Estas voces son utilizadas en el trato cotidiano entre los lunqus; aunque con el transcurso del tiempo se han ido perdiendo, salvo en las zonas rurales, 
https://doi.org/10.46744/bapl.202001.007

donde aún se mantiene el uso de las voces propuestas. Las palabras y acepciones que se presentan fueron recopiladas gracias a colaboradores del distrito de Pocsi, específicamente en el anexo de Tuctumpaya donde, por ser un anexo, aún se usa este léxico con frecuencia. Además, presenta préstamos del quechua, pero no de la información semántica de la palabra en quechua, si no que se produce un cambio semántico, por lo cual, para una mejor compresión de la presente investigación, definimos el término semántica: ciencia que se encarga del estudio del significado de las palabras, esto puede ser tanto su significado connotativo como denotativo. Otro tema que se observa son los cambios semánticos, sobre los cuales Coseriu (1978: 108) afirma: «Lo cambiante es la lengua real en su existir concreto [...] en el hablar de los individuos), esta última concebida como una institución en equilibrio no estático sino dinámico y a la que sólo por exigencia de estudio se imagina detenida». Esto último se observa en el LÉXICO LUNQU, el cual, a pesar de tener escritura parecida al quechua, presenta significados diferentes, los cuales son utilizados en una determinada comunidad.

En cuanto a los estudios realizados sobre el LÉXICO LUNQU, se encuentra el artículo "Arequipeñismos: problemas lexicológicos y lexicográficos» (2018), de Tito Cáceres, en el cual se explican las voces "peculiares» usadas en el habla cotidiana de los arequipeños, y se señala que este léxico es una variedad regional; además, se describen los problemas encontrados al determinar si estas voces provienen del quechua o del aymara, lo cual es hasta ahora tema de debate.

Otro estudio es el Diccionario de arequipeñismos (1999), de Juan Guillermo Carpio Muñoz, en el cual el autor investiga la identidad cultural del mestizaje en la región Arequipa, así como explica lo que es un «arequipeñismo» y las principales características del habla arequipeña; además, presenta la recopilación de algunas voces lunqas o, como él las denomina, «arequipeñismos».

El objetivo propuesto para el presente trabajo es describir los cambios semánticos producidos en el léxico lunqu a partir de los préstamos lexicales del quechua. Para ello, se plantean las siguientes interrogantes: ¿Se han 
producido cambios semánticos en el LÉXICO LUNQU? ¿Qué tipo de cambios semánticos se han producido en el LÉXICO LUNQU?

El trabajo se encuentra estructurado de la siguiente manera: en primer lugar, se presenta un resumen general sobre el tema que se aborda; en segundo lugar, la introducción, en la cual se muestran los antecedentes. Seguido a ello, se muestra la metodología usada para el presente trabajo, en la cual se consigna el planteamiento del problema, asimismo, la justificación y el método de recolección de datos. Luego, se presenta el marco teórico donde citamos a autores que estudiaron la semántica y los cambios semánticos, como Coseriu. En la cuarta parte, se analizan algunas palabras del corpus presentado (Véase anexo 1). Por último, se muestran las conclusiones y las referencias bibliográficas.

\section{Concepto semánticos y contacto lingüístico}

Para un mejor entendimiento, se muestran los siguientes términos que son base para el presente trabajo. Un término importante a entender es semántica. Lyons (1997: 16) afirma que es «el estudio del significado» y lo diferencia de la semántica lingüística, ya que esta última es «[d]el estudio del significado codificado sistemáticamente en el vocabulario y la gramática de las lenguas naturales» (loc cit). Asimismo, la semántica tiene por objeto el estudio «del significado de los signos lingüísticos (ya sean léxicos — palabras_, ya sean oracionales —oración y sintagmas-, ya sean morfológicos — lexemas y morfemas-)» (Rodríguez-Iglesias, 2017: 2).

Otro término importante es cambio semántico, el cual se produce principalmente por factores etnolingüísticos, como lo es el contacto de lenguas. Existen diversas causas por las que se produce el cambio semántico, una de las más comunes es «responder a nuevas necesidades designativas (tales como) la producción de nuevos objetos, el descubrimiento de nuevas realidades o el establecimiento de nuevas pautas de relación social (que) representan situaciones que favorecen el cambio» (Escandell, 2011: 112). 
https://doi.org/10.46744/bapl.202001.007

Por otra parte, se encuentra el término préstamos lingüísticos, los cuales son transferencias de términos que pasan de una lengua a otra. Según Borrego, los préstamos pueden ser comprendidos «en primer lugar mediante deslindes cronológicos e históricos, que como expresión de un cambio viene acompañado de manifestaciones de aculturación que revelan las distintas fases de innovación y de adaptación» (Borrego, 2000: 205).

El léxico lunqu presenta, según su etimología, voces provenientes del quechua, debido al contacto lingüístico, del cual Palacios (s/f.: 1) afirma que "para hablar de consecuencias lingüísticas de las situaciones de contacto, no basta pues con la introducción de algunas palabras [...]; sólo tras un contacto lingüístico prolongado entre dos culturas puede tener lugar la influencia lingüística». Asimismo, Weinreich (1953: 1), pionero en el estudio del contacto lingüístico, señala lo siguiente: «Two or more languages will be said to be in contact if they are used alternately by the same persons.»; por ello, podemos decir que el contacto lingüístico es un proceso social que surge a partir del contacto de culturas; estas, a su vez, al hacer uso de su lengua de forma alternadada, producen cambios lingüísticos.

Como se indicó en el párrafo anterior, el lunqu presenta palabras provenientes del quechua. Respecto al contacto que se da entre estas (quechua y español), se formó el léxico del presente estudio. Callo (2015: 9) señala que «el contacto de lenguas (español-quechua) es un fenómeno donde las influencias entre las lenguas implicadas son recíprocas o bidireccionales, pese a que estos sistemas de comunicación no tienen ningún tipo de parentesco genético ni tipológico». El quechua y el español han estado en contacto permanente desde la llegada de los españoles y, a pesar de que los españoles buscaron imponer su lengua, los quechuahablantes han sabido resistir y han logrado que su lengua perdure hasta nuestros días. El quechua actual presenta una serie de préstamos léxicos, así como de marcas morfosintácticas del español, mientras que el español también presenta marcas características del quechua. Por ello, Callo (2015) señala que se produce una interferencia bidireccional. 


\section{Metodología}

El presente trabajo es de carácter cualitativo, debido a que mediante los datos obtenidos, en este caso una serie de palabras (corpus), se analiza la información para determinar el significado de cada una de estas y, a la vez, se compara con otros diccionarios para, de este modo, establecer qué cambios respecto al significado se produjeron.

El corpus está compuesto por 27 palabras, las cuales se analizan mediante la comparación con otros diccionarios. Para ello, el análisis está estructurado de la siguiente manera: primero, se presenta el significado en el léxico lunqu; luego, se muestra el significado de acuerdo a los otros diccionarios, $y$, al final, mediante los datos obtenidos, se realiza una comparación, en la cual se presentan los rasgos característicos de la palabra y si ha habido variación alguna.

\section{Análisis}

\subsection{Cacha}

En el LÉXICO LUNQU, se define esta acepción de la siguiente manera: cacha. f. Resortera para matar pájaros. No te olvides de llevar la CACHA. (T. V., 48 años.)

González (1608) define cacha como 'mensajero', mientras que en el diccionario de Perroud y Chouvenc (1970) se encuentra la palabra kacha definida como 'mensajero, enviado, criado'. Por último, en el diccionario presentado por el Gobierno Regional del Cusco (2015), se encuentra la palabra q'qacha, la cual es definida como 'espanto, miedo, pavor, impresión de algo/soguilla de cabuya para batir y hacer detonar como una explosión para espantar a los animales dañinos'.

A partir de lo dicho, se puede evidenciar que algunos rasgos semánticos de la palabra en quechua han cambiado para dar paso a la palabra con nueva información semántica en el LÉXICO LUNQU. 
https://doi.org/10.46744/bapl.202001.007

$\begin{array}{cc}\text { QUECHUA } & \text { LUNQU } \\ \text { CACHA } & \text { CACHA } \\ {[+ \text { animado }]} & {[\text {-animado }]} \\ {[\text { +instrumento }]} & {[+ \text { instrumento }]}\end{array}$

La palabra cacha, en quechua, tiene como rasgos principales ser [+animado] y [-instrumento], ya que se refiere a alguien que trasmite o ayuda a enviar cierto mensaje o información a otra persona. En el LÉxICO LUNQU, estas características cambian, aunque mantienen el 'trasmitir o enviar algo', pues una cacha 'resortera' tiene como objetivo enviar piedras pequeñas a un pájaro y así lograr el objetivo de matarlo, por lo que los rasgos señalados cambiarían a [-animado] y [+instrumento].

\subsection{Chaqi}

En el LÉXICO LUNQU se define esta acepción de la siguiente manera: chaqi. $m$. Sopa típica arequipeña preparada principalmente con papas chancadas y tripas de res. Vamos a tomar CHAQI a la plaza. (A. C., 47 años.) I fon. Se pronuncia/cháqe/.

González (1608) define chaqui como 'pie o la pierna', mientras que en el diccionario de Perroud y Chouvenc (1970) se encuentra la palabra chaki definida como 'pie'. Por último, en el diccionario presentado por el Gobierno Regional del Cusco (2015) se encontró la palabra chaki, la cual se define como 'Pie. Pata en animales, y en objetos las partes que sirven de sustentáculo o base'.

A partir de lo dicho, se puede evidenciar que algunos rasgos semánticos de la palabra en quechua han cambiado para dar paso a la palabra con nueva información semántica en el LÉXICO LUNQU.

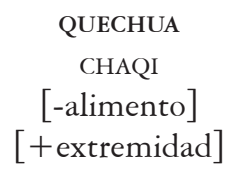

QUECHUA

[-alimento]

$[+$ extremidad $]$

\author{
LUNQU \\ CHAQI \\ $[+$ alimento $]$ \\ [-extremidad]
}


La palabra chaqi, en quechua, tiene como rasgos principales [-alimento] y [+extremidad], entonces, se refiere a una parte del cuerpo, desde la pierna hasta el pie, ya que en la lengua quechua no existe una denominación diferente para el pie y para la pierna, si no que se integran en una misma acepción. En el LÉXICO LUNQU, estas características no se mantienen, al contrario, cambian totalmente y pasa de ser [+ extremidad] a [-extremidad]; aun así, en el chaqi se utiliza como ingrediente principal la carne, y se podría decir que el cambio semántico se produjo a partir de que los lunqus relacionaron la extremidad, o la carne de esta extremidad, con la sopa típica del lugar.

\subsection{Chaqina}

En el LÉXICO LUNQU se define esta acepción de la siguiente manera: chaqina. f. Piedra pequeña del tamaño de la mano usada para freír el cuy o para chancar las papas para el chaqi (LL). Pásame la CHAQINA. (A. C., 47 años.) I Fon. Se pronuncia/chaqéna/.

Gonzalez (1608), define chaquinan como 'camino enxuto de agua o camino sin aguadas o sin fuente', mientras que en el diccionario de Perroud y Chouvenc (1970) se encuentra la palabra chaq definida como 'onomatopeya del ruido de algo que se rompe'. Por último, en el diccionario presentado por el Gobierno Regional del Cusco (2015) no se encontró ninguna palabra similar.

A partir de lo dicho, se puede evidenciar que algunos rasgos semánticos de la palabra en quechua han cambiado para dar paso a la palabra con nueva información semántica en el LÉXICO LUNQU.

$\begin{array}{cc}\text { QUECHUA } & \text { LUNQU } \\ \text { CHAQINA } & \text { CHAQINA } \\ \text {-instrumento }] & {[+ \text { instrumento }]}\end{array}$

La palabra chaqina, en quechua, tiene como rasgo característico [-instrumento], puesto que no se refiere a algo material, sino a un sonido o un camino; sin embargo, se traslada cierta información semántica de la 
https://doi.org/10.46744/bapl.202001.007

palabra en quechua, ya que la acepción 'ruido de algo que se rompe' se puede relacionar a la función que cumple la chaqina, la cual, al ponerse encima del cuy cuando se le está friendo, produce que el pellejo del cuy se quiebre o rompa. Esta información semántica se traslada a chaqina del léxico lunqu, pero, al usar la palabra mencionada para denominar a una piedra, esta adquiere el rasgo [+instrumento].

\subsection{Lluqlla}

En el LÉXICO LUNQU se define esta acepción de la siguiente manera: lluqlla. f.Cauce seco. Ha llovido tanto que hasta por la LLUQLLA ha bajado el agua. (T. V., 48 años.) I Fon. Se pronuncia /llójlla/.

González (1608) define lloclla como 'avenida de agua o diluvio', mientras que en el diccionario de Perroud y Chouvenc (1970) se encuentra la palabra lloqlla definida como 'avenida de agua, ordinariamente turbia, con barro, piedras, etc., torrentera, diluvio'. Por último, en el diccionario presentado por el Gobierno Regional del Cusco (2015) se encontró la palabra lloqlla, la cual es definida como 'aluvión, avenida de lodo, piedras y agua torrentosa/desborde escape de una masa líquida que escurre'.

$\begin{array}{lc}\text { QUECHUA } & \text { LUNQU } \\ \text { LLUQLLA } & \text { LLUQLLA } \\ \text { [ }+ \text { seco }] & {[+ \text { seco }]}\end{array}$

A diferencia de los casos anteriores, la palabra lluqlla no ha tenido gran variación semántica respecto a la palabra de origen, en quechua. Esta palabra tiene como rasgo principal ser [ $\underline{+}$ seco], ya que se refiere al cauce que deja el agua al producirse un aluvión o diluvio. La única variación semántica con el léxico lunqu es que en este lluqlla se refiere a un cauce normalmente seco por el cual no pasa agua ni se mantiene húmedo, en cambio, en quechua, normalmente es entendido como un «camino húmedo». Esta palabra sería un préstamo lingüístico tanto de la palabra base en quechua como de la información semántica de la misma. 


\subsection{Lunqu}

En el LÉXICO LUNQU, se define esta acepción de la siguiente manera: lunqu. com. Arequipeño de rasgos andinos que se dedica a la chacra y al ganado. Solo los LUNQus hablamos así. (A. C., 47 años.) I Fon. Se pronuncia /lónqo/.

González (1608) no define la palabra lunqu, mientras que en el diccionario de Perroud y Chouvenc (1970) se encuentra la palabra longo definida como 'muchacho; lo dicen por desprecio'. Por último, en el diccionario presentado por el Gobierno Regional del Cusco (2015) se encontró la palabra lonq'o, la cual es definida como 'Esfera. Cuerpo'.

A partir de lo dicho, se puede evidenciar que algunos rasgos semánticos de la palabra en quechua han cambiado para dar paso a la palabra con nueva información semántica en el LÉXICO LUNQU.

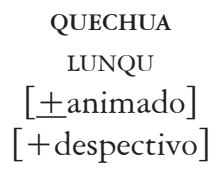

$$
\begin{gathered}
\text { LUNQU } \\
\text { LUNQU } \\
{[+ \text { animado }]} \\
{[\text {-despectivo }]} \\
{[+ \text { rural }]}
\end{gathered}
$$

La palabra lunqu, en quechua, presenta como rasgos principales ser [ \pm animado], [+ despectivo], ya que se usa la palabra para descalificar a alguien respecto a su forma corporal. En cambio, en el léxico lunqu, esta palabra ha adquirido los rasgos [+ animado], [-despectivo] y [+rural], ya que se usa para denominar a los campesinos arequipeños, los cuales gozan de un prestigio en su comunidad. Entonces, aunque sufrió el cambio en estos rasgos, se mantuvo la información semántica en la que se refiere a un sujeto animado.

\subsection{Luqtu}

En el LÉXICO LUNQU, se define esta acepción de la siguiente manera: luqtu. (Del quechua luclu 'huevo podrido'.) adj. Referido a un hue- 
https://doi.org/10.46744/bapl.202001.007

vo de ave: podrido porque no terminó de incubarse. Ese buevo está LUQTU. (T. V., 48 años.) ๆ FON. Se pronuncia /lójto/.

Gonzalez (1608) no define luqtu, mientras que en el diccionario de Perroud y Chouvenc (1970) se encuentra la palabra loqlo definida como 'huevo, hablando de huevo podrido'. Por último, en el diccionario presentado por el Gobierno Regional del Cusco (2015) se encontró la palabra loqt'o, la cual es definida como 'sordera completa'.

A partir de lo dicho, se puede evidenciar que algunos rasgos semánticos de la palabra en quechua han cambiado para dar paso a la palabra con nueva información semántica en el LÉXICO LUNQU.

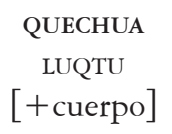

En este caso, al igual que se vio anteriormente, no se produce un cambio semántico, si no que el léxico lunqu tomó la palabra luqtu como préstamo lingüístico.

\subsection{Qala}

En el LÉXICO LUNQU, se define esta acepción de la siguiente manera: qala. com. irón. Persona de la urbe que no se adapta a las costumbres del campo. Ese QALA debe estar sentado en la chacra. (T. V., 48 años.)

González (1608) no define esta palabra en el diccionario, mientras que en el diccionario de Perroud y Chouvenc (1970) se encuentra la palabra jala definida como 'desnudo, pelado, limpio'. Por último, en el diccionario presentado por el Gobierno Regional del Cusco (2015) se encontró la palabra qala, la cual es definida como 'Desnudo, despojado de todo vestido'. 
A partir de lo dicho, se puede evidenciar que algunos rasgos semánticos de la palabra en quechua han cambiado para dar paso a la palabra con nueva información semántica en el LÉXICO LUNQU.
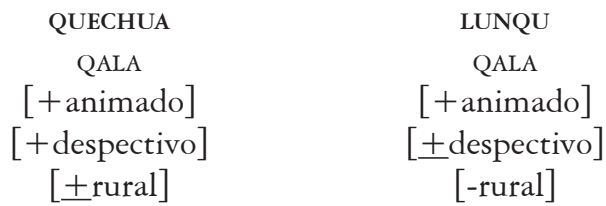

La palabra qala presenta como rasgos principales ser [+ animado] y [+despectivo], el primer rasgo se trasladó a la palabra en el léxico lunqu, pero varió semánticamente el rasgo [ $\alpha$ despectivo], puesto que en este léxico, generalmente, no se utiliza la palabra para denigrar a alguien. El traslado de la carga semántica se hizo en un sentido metafórico, puesto que el qala, al no adaptarse a las costumbres de los lunqus, estaría desnudo, sin la «vestimenta» lunqu. Respecto al rasgo [rural], puede usarse el término en quechua para denominar a cualquier persona, sin importar su procedencia o costumbres; en cambio, en el léxico lunqu, solo se denomina así a una persona que no sea rural.

\subsection{Qulloto, ta}

En el LÉXICO LUNQU, se define esta acepción de la siguiente manera: qulloto, ta. adj. 1. Que le falta un dedo. No me gusta verte tu mano QULLOTA. (A. C., 47 años.) || 2. adj. Que le falta rabo. Ese perro QULLOTocome demasiado. (T. V., 48 años.) || 3. f. Gallina que tiene rabo pequeño. Trae una QULLOTA para el caldo. (T. V., 48 años.) ๆ FON. Se pronuncia /qollota/.

González (1608) no registra la palabra qulloto, mientras que en el diccionario de Perroud y Chouvenc (1970) se encuentra la palabra jollota definida como 'batán pequeño, piedra redonda para moler ahí. Manco (jollota maki)'. Por último, en el diccionario presentado por el Gobierno Regional del Cusco (2015) se encuentra la palabra qollota, la cual es definida 
https://doi.org/10.46744/bapl.202001.007

como 'Esfera, bola de piedra usada para moler condimentos y medicina, estrujándolas dentro de un mortero'.

A partir de lo dicho, se puede evidenciar que algunos rasgos semánticos de la palabra en quechua han cambiado para dar paso a la palabra con nueva información semántica en el LÉXICO LUNQU.

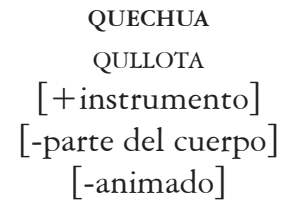

La palabra qullota, en quechua, presenta los rasgos característicos [+instrumento], [-animado], pero estos rasgos cambian en el léxico lunqu y se convierten en [-instrumento], [+ parte del cuerpo] y [+ animado]. La información semántica que se traslada del quechua al lunqu es la que involucra la función del batán, el cual 'muele condimentos'. Al decir que muele nos referimos a que algo sólido se 'desmenuza en partes', lo que puede ser relacionado con el rasgo [parte del cuerpo], ya que qullota significa que a alguien le falta una parte del cuerpo, como la mano o el rabo, lo que lo relaciona así con la información semántica del verbo moler.

\section{Conclusiones}

El contacto lingüístico entre el español y el quechua permitió que, con el pasar de los años, las palabras en quechua evolucionaran históricamente, perdieran rasgos de la palabra base y adquirieran nuevas características. Esto produjo el cambio léxico-semántico. El léxico lunqu registra préstamos lingüísticos del quechua, pero estas palabras no contienen la misma información semántica que la palabra original. La mayor parte de los sustantivos del léxico lunqu presentan cambios semánticos, donde uno de los rasgos de la palabra en quechua se traslada tal cual a la palabra lunqu, o los principales rasgos de la palabra base cambian totalmente.

Bol. Acad. peru. leng. 67(67), 2020 / e-ISSN: 2708-2644 


\section{BIBLIOGRAFÍA}

ASOCIACIÓN DE ACADEMIAS DE LA LENGUA ESPAÑOLA (2010). Diccionario de americanismos. Madrid: Santillana.

CÁCERES, T. (2018). Ensayos de literatura arequipeña. Lima: UNAS.

CALLO, D. (2015). Interferencia gramatical en el quechua de bablantes bilingües del valle del Colca. Tesis para optar por el grado de Doctor en Lingüística. Lima: UNMSM.

COSERIU, E. (1978). Gramática, semántica, universales. Madrid: Gredos

ESCANDELL, V. (2011). Apuntes de semántica léxica. Madrid: UNED.

GOBIERNO REGIONAL DEL CUSCO. (2005) Diccionario quechua español - quechual qheswa - español - qheswalsimi taque. Gobierno Regional del Cusco.

GONZALES, D. (1608). Vocabulario de la lengua general de todo el Perú llamada lengua qquichua o del inca.

KATZ, J. y FODOR J. (1976). La estructura de una teoría semántica. México: Siglo Veintiuno editores.

PALACIOS, A. (s/f.). Situaciones de contacto lingüístico en Hispanoamérica: español y lenguas. Madrid: Universidad Autónoma de Madrid.

PERROUD, Pedro y CHOUVENC, J. (1970). Diccionario castellano/kechwal castellano. Lima: Semanario San Alfonso. Padres redentoristas.

REAL ACADEMIA ESPAÑOLA (2014). Diccionario de lengua española. Madrid: RAE.

RODRIGUEZ-IGLESIAS, I. (2007). Semántica y significado. Concepción heptagonal y relaciones semánticas. Barcelona: Revista de 
https://doi.org/10.46744/bapl.202001.007

Investigación Científica sobre Lenguaje, Sociedad y Comunicación.

WEINRECIH, U. (1953). Languages in contact, findings and problems. New York: Linguistic Circle of New York.

\section{ANEXO 1}

Para el presente trabajo, se contó con la información brindada por tres colaboradores: Tomas Vilca Ynfantes, Angelita Coaguila Vilca y Norma Vilca Ynfantes. Esta información se obtuvo a partir de conversaciones realizadas de manera cotidiana.

\begin{tabular}{|c|c|c|c|}
\hline $\mathbf{N}^{\circ}$ & Nombres & Abr. & Edad \\
\hline 1 & Vilca Ynfantes, Tomás & TV & 48 años \\
\hline 2 & Coaguila Vilca, Angelita & AC & 47 años \\
\hline 3 & Vilca Ynfantes, Norma & NV & 52 años \\
\hline
\end{tabular}




\section{LISTADO DE PALABRAS DEL LÉXICO LUNQU}

Cacha

Caqllado

Chaqi

Chaqina

Chuqñi

Culoqala

Lluqlla

Lunqu

Macurci

Misciriche

Mocotiqti

Pallapar
C

M

$\begin{array}{ll} & \text { Saqra } \\ \mathbf{P} & \text { Taqpi } \\ & \text { Tiwayar } \\ & \text { Tuqpir }\end{array}$

Q

Qachir

Qala

Qaramozo

Qincha

Qinchoso

Qipu

Qirqu

Qiti

Qulloto, ta

Qurito

S

$\mathrm{T}$ 